\title{
Microarrays in Glycoproteomics Research
}

\author{
Tingting Yue, BS [Research Associate, Doctoral Candidate] and \\ Van Andel Research Institute, 333 Bostwick NE, Grand Rapids, MI 49503, Cell and Molecular \\ Biology Program, Michigan State University, East Lansing, MI
}

Brian B. Haab, PhD [Senior Scientific Investigator]

Van Andel Research Institute, Grand Rapids, MI

Tingting Yue: Tingting.yue@vai.org

\begin{abstract}
Microarrays have been extremely useful for investigating binding interactions among diverse types of molecular species, with the main advantage being the ability to examine many interactions using small amount of samples and reagents. Microarrays are increasingly being used to advance research in the field of glycobiology, which is the study of the nature and function and carbohydrates in health and disease. Several types of microarrays are being used in the study of glycans and proteins in glycobiology, including glycan arrays to study the recognition of carbohydrates, lectin arrays to determine carbohydrate expression on purified proteins or on cells, and antibody arrays to examine the variation in particular glycan structures on specific proteins. This review will cover the technology and applications of these types of microarrays, as well as their use for obtaining complementary information on various aspects of glycobiology.
\end{abstract}

\section{Keywords}

Glycobiology; glycoproteomics; glycan microarrays; lectin microarrays; antibody microarrays

\section{Introduction}

The importance of carbohydrate post-translational modifications on proteins has become increasingly clear over many decades of advances in our understanding of glycobiology. It is now widely appreciated that the carbohydrate side chains play critical roles in the structure and function of many, if not most, cell surface and secreted proteins. These roles include the guiding of proper protein folding, the maintenance of protein conformation, mediating receptor-ligand and protein-protein interactions, providing biophysical polarity or hydrophilicity, and guiding immune recognition [1]. Abnormal glycosylation also is associated with a variety of inherited and sporadic diseases [2,3], testifying to the necessity of proper glycosylation for the maintenance of health. Furthermore, glycosylation has been found to be an integral part of the proper functioning of every organism in nature. Given this broad importance of glycobiology, the field has increasing importance in applied research in biotechnology and biomedicine. For example, treatment strategies based on interfering with

Correspondence to: Brian B. Haab, Ph.D., Van Andel Research Institute, 333 Bostwick NE, Grand Rapids, MI 49503, Brian.haab@vai.org, Tel. 616-234-5268, Fax 616-234-5269.

Publisher's Disclaimer: This is a PDF file of an unedited manuscript that has been accepted for publication. As a service to our customers we are providing this early version of the manuscript. The manuscript will undergo copyediting, typesetting, and review of the resulting proof before it is published in its final citable form. Please note that during the production process errors may be discovered which could affect the content, and all legal disclaimers that apply to the journal pertain. 
glycan-mediated process or targeting cancer glycans are under development [4], and bloodbased diagnostic tests using glycan detection may be possible [2].

Protein glycosylation refers to the chains of monosaccharide building blocks that are covalently linked to particular amino acid residues (usually serine, threonine, or asparagine residues). The monosaccharides are often five- or six-carbon cyclic structures with various modifications and isometries. Glycosidic bonds join the monosaccharides at any of the carbons with either an 'alpha' or a 'beta' linkage-referring to the stereoisometry of the linkage at a chiral carbon - and in a linear or branched fashion. This variety in the components and the linkages results in a huge diversity of structures that can be formed. In reality, although great diversity indeed is observed in each organism, particular motifs and structural themes are prevalent, bringing some order to the complexity.

The study of carbohydrate biology has been viewed as daunting by many researchers due to the heavy chemistry emphasis and perceived difficulty in the traditional analytical methods. Indeed, historic glycobiology has focused primarily on carbohydrate chemistry and involved techniques not typically included in the education of biologists. However, the development of modern biological methods provided new ways of studying glycobiology, resulting in huge advances in our understanding of the genetic and biochemical basis of glycan synthesis and the molecular and cellular biology of glycans. The new tools for studying glycobiology make the field more accessible and useful to a broader base of researchers- tools such as glycan-binding antibodies and proteins, genetically-modified cells and organisms, gene expression profiling of glycan-related genes, and advanced chromatographic and massspectrometry analysis of glycan structures.

Another set of tools that is advancing glycobiology is built on the microarray platform. Microarray methods analyzing RNA and DNA transformed gene expression and genetic research following their introduction in the early 1990s [5]. The usefulness of the microarray platform lies in its multiplexing capability—enabling the acquisition of many data points in parallel —and its miniaturization—resulting in very small consumption of reagents and samples. These benefits were recognized by researchers studying other molecule types, including proteins, antibodies, lipids, and glycans, but microarrays for such studies developed more slowly due to increased technical difficulty. Currently, microarrays for studying all types of molecules are becoming established and broadly applied.

This article deals with the application of microarray formats to the study of glycoproteins and glycans. We survey the technology and applications of three different types of microarrays developed for glycoproteomics research: glycan microarrays, lectin microarrays, and antibody-lectin sandwich microarrays (fig. 1). Each format is used in distinct and complementary types of experiments and has facilitated advances in glycobiology research. The review focuses on glycans and proteins and therefore will not cover DNA arrays, such as the Glyco-gene DNA chip (provided by the Consortium for Functional Glycomics) for profiling the expression of genes involved in the glycosylation machinery.

\section{Glycan microarrays}

A major goal in glycobiology research is to probe and characterize interactions between glycans and various types of glycan-binding proteins. Conventional methods for such studies are not suitable for the profiling of many different glycans ("glycomics" studies). For example, the glycan-binding specificities of lectins have been effectively probed by determining the elution profiles of various glycans in affinity chromatography [6], but these experiments require significant amount of glycan material per test with each glycan tested 
sequentially. Likewise, enzymatic studies on glycans can only be performed on a single glycan per assay and require significant material.

The glycan microarray addresses these limitations by enabling binding analyses to many different carbohydrate structures in small sample volumes and with minimal consumption of the reagents. The low consumption of the carbohydrate structures is particularly important because of the difficulty and time required to synthesize or isolate those structures. Below we present an overview of the types of carbohydrate microarrays that have been produced, follow by a review of the applications of carbohydrate microarrays.

\section{Technological overview}

Several different demonstrations of carbohydrate microarrays appeared in 2002 with a variety of fabrication techniques [7, 8]. Houseman et al. used covalent attachment of carbohydrate-cyclopentadiene conjugates to self-assembled monolayers on gold surfaces to create monosaccharide arrays, and these chips were used to test interactions with selected lectins and enzymes [9]. Mono- and di-saccharide chips were created by Park and Shin [10] using the covalent attachment of maleimide-linked carbohydrates to thiol-derivatized slides. Fazio et al. attached oligosaccharides to hydrocarbon chains, which were then attached in a non-covalent fashion to the bottoms of wells of polystyrene microtiter plates [11]. These oligosaccharides were probed with lectins and also were modified directly in the plate using glycosyltransferases. Wang et al. used non-covalent attachment to create glycan microarrays, by spotting carbohydrate-containing macromolecules onto nitrocellulosecoated glass slides [12]. A wide variety of microbial antigens were probed with anticarbohydrate antibodies. Fukui and colleagues also used a nitrocellulose surface, onto which they spotted lipid-linked oligosaccharide probes generated from glycoproteins and polysaccharides [13]. Non-covalent attachment also was used by Willats and coworkers, in which complex polysaccharides, proteo-glycans, and neo-glycoproteins were spotted onto oxidized polystyrene, followed by probing with anti-glycan antibodies [14]. Several of the applications above show the value of oligosaccharides derived from biological sources, which represent complex, biologically-relevant structures that are not able to be synthesized. Xia et al. also demonstrated a practical method for the generation of such probes [15]. The above demonstrations primarily used fluorescence scanning of dye-labeled probes for detection.

A significant advance in the utility and availability of glycan microarray technology came through the development of glycan microarrays by the Consortium for Function Glycomics (CFG). The CFG is dedicated to providing resources, technology and collaborative opportunities for investigators focused on carbohydrate-related research. Researchers from the CFG synthesized over 200 biologically-relevant glycans attached to amine-conjugated spacers, and spotted them onto NHS-activated glass slides to form covalent linkages [16]. The arrays were initially used to characterize specificities of plant lectins, human lectins, glycan-binding antibodies, and bacterial and viral proteins [16]. Since then, the CFG has profiled the specificities of hundreds of glycan-binding proteins for researchers participating in the consortium, and the data are made available through the CFG website. Some of the applications of these and other glycan microarray platforms are described below.

\section{Determining specificities of glycan-binding proteins}

The most widely-used application of glycan microarrays is to characterize the specificities of glycan-binding proteins and antibodies. An important class of molecules for which glycan-binding specificity has been studies is lectins, which are non-enzymatic, glycanbinding proteins found in all types of organisms. Lectins play roles in diverse processes such as cell migration, immune recognition, and angiogenesis, among others [17], and 
information about lectin binding specificities gives important clues about function. In addition, the characterization of lectin specificity is helpful for their use as analytical reagents.

Manimala et al. [18] developed a glycan microarray containing 54 conjugated glycans, ranging from basic monosaccharides to more complicated cancer associated glycan motifs, to obtain the binding profiles of 24 lectins. Glycan microarray slides were incubated with biotinylated lectins in serial dilutions and probed with streptavidin-horseradish peroxidase. The authors identified specificities that were not previously observed due to the lack of a practical approaches for screening that many interactions. For example, a similar study using the conventional ELISA method would require the use of over 100-fold more sample.

Glycan microarrays also have been useful to characterize the specificities of monoclonal antibodies raised against carbohydrates. Microarrays containing 80 different carbohydrates were used by Manimala and coworkers to study the specificities of 27 different anti-glycan antibodies, which showed that most antibodies bound other glycans in addition to their nominal targets [19]. This finding may reflect the difficulty in generating truly specific antiglycan antibodies. The same group also showed that antibodies raised against the $\mathrm{Tn}$ antigen (GalNAca1-O-Ser/Thr), an important epitope in cancer and microbiology, also reacted with the blood group A structure (a related glycan), which has implications for diagnostics using anti-glycan antibodies [20]. In related applications, the glycan-binding specificities of antibodies raised against the cell wall polymers from the plant Arabidopsis thaliana were characterized using arrays containing 50 cell wall glycans [21], and specialized microarrays were designed to characterize the specificities of monoclonal antibodies targeting the Globo $\mathrm{H}$ hexasaccharide found on cell surfaces from breast, prostate, and ovarian cancers [22].

\section{Anti-glycan immune responses characterized using glycan arrays}

Certain glycan structures can elicit an immune response if the structures are not normally presented to the host immune system. Indeed, the blood group glycan structures of the ABO system elicit antibodies against structures not found in the host, which can lead to the agglutination of red blood cells from unmatched blood. Anti-glycan antibodies also can be generated in immune responses against pathogens and cancer. The glycan array provides a powerful tool for probing for anti-glycan antibodies and for determining their specificities. In the work of Lawrie et al. [23], a glycan microarray containing 37 covalently bound glycans was used to identify carbohydrates that trigger humoral immune responses in classical Hodgkin's lymphoma (cHL) patients. Total IgG and IgM from groups of patient and age/sex matched control individuals were purified, pooled within the same diagnostic states, and applied to indivdual arrays. Biotin-conjugated anti-human $\operatorname{IgG}$ or $\operatorname{IgM}$ antibodies, followed by dye-labeled steptavidin and fluorescence scanning, were used to detect antiglycan antibodies. Antibodies against five carbohydrates, including the $\mathrm{T}$ and $\mathrm{Tn}$ antigens (Galß1,3GalNAca- and GalNAca-, respectively) were identified from cHL patients and became targets for further investigation.

In another cancer-related study, glycan microarrays were used by Wang et al. to show that breast cancer patients have an increased level of antibodies targeting a glycan structure called Globo H, which is found on the surfaces of breast cancer cells [24]. Globo H and its truncated analogs were robotically spotted at various concentration onto NHS-coated glass slides for covalent attachment, and bound antibodies were detected by Cy3-labeled anti-IgG secondary antibodies. The detection of anti-Globo $\mathrm{H}$ antibodies may be useful for breast cancer diagnostics.

Antibodies against bacterial pathogens also were detected using carbohydrate arrays. Arrays containing glycans from anthrax toxin ( $B$. anthracis) were generated to characterize 
antibodies from rabbits that had been infected with anthrax [25]. Antibodies were found that reacted with a prominent tetrasaccharide specifically found on the spore surface, which confirms this structure as an important immunological target. A carbohydrate microarray containing oligosaccharides specific for different versions of Salmonella enterica was used to identify glycan-reactive antibodies and to confirm the presence of strain subgroups in patients suffering from salmonellosis [26]. This method might be useful for rapid diagnosis or for epidemiological or vaccine studies.

\section{Microbiology applications}

Glycans play important roles in the recognition and attachment to host sites by microbial pathogens. In most cases the nature of the glycan attachment sites is not known. Glycan microarrays have been useful for studying that question. Stevens et al. [27] used glycan microarrays to investigate the host specificity of influenza viruses. Glycans with different carbohydrate components or glycosidic linkages printed in the microarray were incubated with recombinant hemagglutinins from membranes of various influenza virus strains or hybridized directly with whole virus. Different virus strains were found to have distinct preferences of binding to particular glycosidic linkage types. Profiling of strain-specific glycoprotein binding specificity from various virus strains using glycan microarray could provide crucial information in understanding virus adaptation and species barriers, which may be useful for preventing human infection.

Disney et al. [28] used glycan microarrays containing five different monosaccharides to detect pathogens and test for their antibiotic susceptibility. Microarray slides were hybridized with $E$. coli cells that had been labeled with a nucleic acid staining dye. After washing away unbound bacteria, slides were scanned to detect fluorescence, indicating the glycan-bacteria binding. Mutant strains with altered carbohydrate binding patterns could be detected from this assay. The authors proposed that a carbohydrate-binding "fingerprint" identified using this glycan microarray can be used to determine the types of bacteria present within a complex mixture. Since this is a nondestructive method, bacteria captured on the arrays can further be harvested and tested for antibacterial susceptibility, which is not possible using traditional destructive methods, such as those requiring PCR.

\section{Enzymatic studies}

Another important application of glycan microarrays is to evaluate the specificities and activities of sugar-processing enzymes, such as transferases used for the addition of carbohydrate units [29-31]. Increased knowledge about glycosyltransferases and glycosidases would lead to a better understanding of how certain glycan chains are produced and how to use these enzymes for the synthesis of carbohydrates. Several groups have demonstrated the modification of sugars immobilized in arrays. In the work of Park et al. [30], the treatment of glycan arrays with UDP-Gal and $\beta$-1,4-galactosyltransferase (GalT) resulted in the conversion of $\mathrm{N}$-acetylglucosamine (GlcNAc) to lactosamine only in the absence of fucose on the GlcNAc, thus providing valuable information about the enzyme specificity. In another experiment from the same group, the authors successfully synthesized Sialyl Le ${ }^{\mathrm{x}}$ (NeuNAca 2,3Gal $\beta 1,4$ (Fuca 1,3)GlcNAc) from arrayed GlcNAc by the addition of a series of glycotransferase and sugar units [29]. Their work showed the efficiency and potential of using glycan microarray to characterize carbohydrate-processing enzymes as well as the possibility of enzymatic transformation from simple glycans to complex carbohydrates directly on the array. In more recent work, the acceptor specificities of multiple sialyltransferases were compared, showing differences in substrates between human and rat sialyltransferases [31]. 


\section{Lectin microarrays}

Lectin microarrays also take advantage of the low-volume and multiplexing capabilities of microarrays, but provide complementary information to glycan microarrays. Lectins were first recognized by their ability to agglutinating red blood cells [32], and later the term "lectin" was adopted when it was realized that there existed a class of carbohydrate-binding proteins [32]. Although lectins were originally isolated from plants, they were later found ubiquitously in nature [33]. Lectins originally were classified according to their glycanbinding specificities, but they are now more consistently grouped according to sequence and structural motifs [34].

Plant lectins have become extremely valuable analytical tools for the detection of particular carbohydrate structures. As affinity reagents, lectins can detect glycans with high reproducibility and in a variety of formats, and thus provide a good alternative to other glycan detection methods involving enzymatic digestion, chromatography or mass spectrometry, which have low reproducibility and low throughput. The specificity of detecting particular glycans depends on the lectin used; some are highly specific, and others have broad specificities. Lectin detection can have good sensitivity due to multi-valent binding, resulting from either a multi-subunit or multiple carbohydrate-binding sites within a single polypeptide [35].

Lectins have long been used for detection and purification of glycans in various research fields [36]. Lectins have been used extensively in immunohistochemistry, for example in studies to examine the tissue distribution in pancreatic tumors of certain blood-group carbohydrates [37, 38]. Lectins also have been used in immuno-affinity electrophoresis and blotting methods, for example to identify cancer-associated glycan variants on the serum proteins a-fetoprotein [39], haptoglobin [40, 41], a-1-acid glycoprotein [42], and a-1antitrypsin [43]. Although lectins have been used as sugar detection reagents for decades [17], the use of multiple lectins for the analysis of biological samples was not common due to the amount of material and time required for each assay.

\section{Technological overview}

The lectin microarray made it practically feasible to obtain glycan measurements on a given sample from multiple, different lectins. By incubating samples on an array of lectins and determining the amount of binding to each lectin, a broad profile of the glycans present in the sample can be rapidly obtained with minimal sample consumption. This approach has many advantages over standard methods of glycan analysis, such as reduced cost, time, and sample consumption, with increased reproducibility. An additional advantage is that lectins can provide information about linkages between monosaccharides (for example whether the alpha or beta configuration), which is not discernable using mass-spectrometry analysis.

Some challenges accompany the use of lectin microarrays. One is the question of specificity. Lectins cannot give exact structural information, but rather mainly provide information about terminal structures. Moreover, the specificities of some lectins are incompletely understood and may involve more than one particular structure. Some have suggested that through the use of many lectins, the analysis of an overall lectin-binding profile can overcome some of the ambiguities associated with lectin binding [44], although the effectiveness of such a strategy has not been demonstrated. Another challenge can be detection sensitivity, since lectin-glycan interactions are weak compared with DNA-RNA or antibody-antigen hybrids. Standard washing protocols developed for protein or DNA microarrays may result in the significant loss of binding. An evanescent-field fluorescence strategy is a promising solution [45], since it allows the sensitive, real-time observation of monovalent lectin-oligosaccharide interactions under equilibrium conditions without the 
requirement of washing. The optimization of this method enabled the detection of glycoproteins down to a $100 \mathrm{pM}$ concentration [46].

The initial demonstrations of lectin microarrays $[47,48]$ used standard arraying methods that had been developed for DNA and protein microarrays. Lectins were printed on aldehyde- or epoxide-derivatized glass slides to achieve covalent immobilization, or were linked via biotin-streptavidin bridges on photoactivatable dextran-coated slides. Another lectin array used the non-covalent suspension of lectins in an aqueous hydrogel matrix [49], which may better preserve the conformation and activity of lectins relative to binding on planar surfaces. The arrayed lectins were hybridized with samples (e.g. glycoproteins) that were directly labeled with a fluorescent tag or that were recognized by a labeled detection reagent.

\section{Studies of protein glycosylation}

The major application of lectin microarrays has been to rapidly investigate the glycosylation of purified glycoproteins which were incubated on the arrays. For example, Kuno and coworkers used arrays containing 39 lectins to detect glycosylation differences between various glycoproteins and changes in glycosylation induced by treatment with glycosidases [45]. The incubation of purified proteins, as opposed to mixtures of proteins, is important to simplify the interpretation of the data, so that one may know the identity of the protein binding each lectin. However, others have demonstrated the incubation of complex mixtures of proteins onto lectin arrays, thus achieving a summary view of a cell "glycome." Pilobello and coworkers used a ratiometric approach to examining changes in bacterial cell-surface glycomes [50]. Isolated membrane proteins from two bacterial cultures were differentially labeled with Cy3 and Cy5 fluorescent dyes and co-incubated on arrays containing up to 58 different lectins. The $\mathrm{Cy} 3 / \mathrm{Cy} 5$ ratio at each spot provided a sensitive indicator of differences between the cultures and allowed for normalization between arrays. This analysis enabled the observation of glycosylation changes occurring in response to cell differentiation. The evanescent-field fluorescence method mentioned above also was applicable to the study of crude glycoproteins extracted from mammalian cells [51]. While the approach of incubating multiple proteins on lectin arrays offers a summary view of the glycan structures on a cell, it has the disadvantage of integrating information from all proteins, so that glycan changes that occur only on a subset of proteins may be lost in a background of non-changing proteins.

\section{Cell surface glycosylation}

A potentially simpler and more direct view of cell-surface glycosylation has been achieved by incubating live cells on the surfaces of lectin microarrays. The use of whole cells as opposed to cell extracts has an advantage of preserving higher-order structures, which may be biologically significant and important for lectin binding. Early work by Zheng et al. [52] used covalent immobilization of lectins on self-assembled monolayers that were functionalized with NHS. Cultured cells were incubated on the spotted lectins, and the binding of the cells to the lectins was visualized with an inverted microscope. The gold base substrate was thin enough to allow the imaging. The authors showed differences in the glycosylation of the two cell types. In later work by the same group [53], the authors used this technology to explore glycan differences between normal and breast cancer cell lines. Significant variation in glycosylation was identified which correlated with metastatic potential as well as metastatic location preference.

Lectin microarrays also were used to examine dynamic changes to $E$. coli bacterial glycosylation [54]. The bacteria were labeled with a dye that binds to DNA to allow detection by fluorescence after incubation on the arrays. The authors could distinguish $E$. coli strains based on glycosylation and could observe growth-dependent variation in glycosylation on particular strains. Lectin arrays employing evanescent-field fluorescence, 
as described earlier [45], were used to examine dynamic changes to the cell surfaces glycomes of mammalian cells that had been fluorescently labeled with a DNA-binding dye [55]. Alterations in lectin-binding patterns were seen in glycosylation-defective mutants of $\mathrm{CHO}$ cells and in splenocytes from mice with a genetic knockout of a glycosyltransferase gene. Changes in cell surface glycosylation associated with erythroblast differentiation also were observed. Another study using arrays of 94 lectins and a similar detection method examined the lectin-binding signatures of 24 different human cell lines and predicted functional phenotypes based on lectin-binding profiles [56].

\section{Antibody-lectin sandwich microarrays}

Another array-based glycoproteomics method is the antibody-lectin sandwich microarray. The value of antibody-lectin sandwich microarrays for glycoproteomics studies is that they provide precise measurements of glycan levels on specific proteins captured directly from biological samples. This capability enables detailed views of how glycans on particular proteins change in association with disease states or sample conditions. Previous methods did not practically allow that type of investigation. Studies employing enzymatic, chromatographic, and mass spectrometry methods have been very effective for providing detailed information about glycan structures in individual samples, but due to high sample consumption, low throughput, or low reproducibility, such studies did not reveal how frequently particular glycans on particular proteins appear, how closely they are associated with particular disease states, or the distribution of protein carriers on which they appear. Affinity-based methods, using reagents such as lectins or glycan-binding antibodies to detect glycans, can provide that information, because one may reproducibly measure the glycan levels over multiple samples. While affinity-based glycosylation studies do not provide the structural detail provided by mass spectrometry and enzymatic methods, they can provide information about the biological variation of a particular motif.

The method starts with an antibody microarray-essentially identical to those developed for multiplexed protein analyses [57] (fig. 1). The antibodies on the array can be chosen to target various glycoproteins. A complex biological sample is incubated on the array, resulting in the capture of glycoproteins by the antibodies. The next step is to probe the glycans on the captured proteins using labeled lectins. The amount of lectin binding at each capture antibody indicates the amount of a particular glycan on the proteins captured by each antibody. A variety of lectins could be used on a given sample in order to probe several different types of glycans. Glycan-binding antibodies also could be used as detection reagents, such as those raised against the Thomsen-Friedenreich antigens [58] or the Lewis blood-group structures [59]. Antibody-lectin sandwich arrays are similar to previous approaches using lectins in the capture or detection of proteins in microtiter plates [60], but they harness the power of microarrays to provide high information content in low sample volumes.

In order to properly interpret the amount of glycan on a protein, one must also know the underlying protein concentration. That complementary information may be conveniently obtained using antibody microarrays in a standard sandwich assay format to detect core protein levels (fig. 2). Therefore a sample may be incubated multiple times on replicate microarrays, each time probed with a different lectin, to characterize glycan levels (fig. 2b), or with antibodies, to characterize protein levels (fig. 2a). A previous study [61] showed the value of using both formats to detect glycosylation differences between samples (fig. 2c).

The ability to probe each sample multiple times, and to probe many samples (as would be required for clinical studies), requires the ability to run many samples efficiently and to consume small sample volumes in each assay. A practical method for the high-throughput 
processing of low-volume microarrays was demonstrated earlier [62]. Multiple, replicate microarrays are printed onto a microscope slide, and the arrays are separated from one another by hydrophobic, wax borders that are precisely imprinted onto the slide. The borders are imprinted using a device (The Gel Company, San Francisco, CA) that elevates a stamp out of a wax bath, which sits atop a hotplate to melt the wax, to contact a microscope slide suspended above the wax bath (fig. 3a). The wax borders prevent liquid from spilling from one array to another, and they remain on the slide throughout the processing steps and the fluorescence scanning (fig. 3b). Any size or pattern of arrays could be accommodated by using the appropriate stamp (fig. 3c). A high-throughput strategy using this format is to incubate sets of samples in a randomized order on a microscope slide and then probe the captured proteins on the slide with a lectin (fig. 3d). Such a strategy has been used for highthroughput antibody array processing in multiple studies [61, 63, 64].

Ongoing studies are applying this technology in various ways. One application is the profiling of glycan changes on particular proteins in various disease states, to identify those most associated with disease and to develop new biomarkers. Unpublished data in our lab shows that certain glycans on particular serum proteins are altered in pancreatic cancer more frequently than the underlying core protein. Because of that relationship, the measurement of the glycan on the protein performs better as a biomarker than the measurement of the protein. The same study also provides information on the prevalence of glycan alterations in various patient populations, which was not known before because of the aforementioned limitations of conventional glycobiology methods. Another application of this technology is the study of glycan changes induced by various perturbations to cultured cells. For example, we have examined the effects of pro-inflammatory stimuli on the glycan structures of mucins secreted by cancer cells. We found that the induced glycan structures are similar to those observed in clinical samples from pancreatic cancer patients, suggesting that cancerassociated glycans can arise in response to a pro-inflammatory tumor microenvironment. These types of studies show how the capabilities of antibody-lectin sandwich arrays, such as the ability to measure specific glycans on multiple, specific proteins captured from biological samples, and the ability to precisely measure changes between samples in those glycan levels, enable access to information that would be difficult to acquire using conventional methods.

\section{Summary}

The above overview provides insights into how microarray platforms are stimulating advances in glycoproteomics research. Each of the platforms discussed here can be used in multiple types of experiments to produce distinct types of information. The many different areas of glycobiology in which glycan, lectin, and antibody microarrays have been applied testify to the versatility of the platforms. Glycan arrays are valuable for studying protein and cell binding to glycan structures, and they have been used to study glycan-binding specificities, disease-associated anti-glycan antibodies, microbe-carbohydrate interactions, and the activities of glycan-processing enzymes. Lectin arrays provide a convenient and rapid tool for assessing the overall glycan content of a sample. These tools have facilitated studies on the glycosylation of purified proteins and complex mixtures of proteins as well the total carbohydrate content of cell surfaces. Finally, antibody-lectin sandwich microarrays are an additional tool for glycoproteomics, and provide information about the carbohydrate on multiple, distinct proteins captured directly from biological samples. This tool enables views of glycan variation on specific proteins in patient populations or in response to changing conditions. All of these tools can provide experimental information that was not obtainable using conventional technologies. Therefore, the increased usage of these approaches is anticipated to drive further major advances in glycoproteomics. 


\section{Acknowledgments}

This work was supported by the NCI (grant R33 CA122890) and the Van Andel Institute.

\section{References}

1. Varki, A.; Cummings, R.; Esko, J.; Freeze, H.; Hart, G.; Marth, J. Essentials of glycobiology. Cold Spring Harbor, NY: Cold Spring Harbor Laboratory Press; 1999.

2. Dube DH, Bertozzi CR. Glycans in cancer and inflammation--potential for therapeutics and diagnostics. Nat Rev Drug Discov. 2005; 4(6):477-488. [PubMed: 15931257]

3. Dennis JW, Granovsky M, Warren CE. Glycoprotein glycosylation and cancer progression. Biochim Biophys Acta. 1999; 1473(1):21-34. [PubMed: 10580127]

4. Fuster MM, Esko JD. The sweet and sour of cancer: glycans as novel therapeutic targets. Nat Rev Cancer. 2005; 5(7):526-542. [PubMed: 16069816]

5. Schena M, Shalon D, Davis RW, Brown PO. Quantitative monitoring of gene expression patterns with a complementary DNA microarray. Science. 1995; 270(5235):467-470. [PubMed: 7569999]

6. Hirabayashi J, Hashidate T, Arata Y, et al. Oligosaccharide specificity of galectins: a search by frontal affinity chromatography. Biochim Biophys Acta. 2002; 1572(2-3):232-254. [PubMed: 12223272]

7. Culf AS, Cuperlovic-Culf M, Ouellette RJ. Carbohydrate microarrays: survey of fabrication techniques. Omics. 2006; 10(3):289-310. [PubMed: 17069509]

8. Wang D. Carbohydrate microarrays. Proteomics. 2003; 3(11):2167-2175. [PubMed: 14595816]

9. Houseman BT, Mrksich M. Carbohydrate arrays for the evaluation of protein binding and enzymatic modification. Chem Biol. 2002; 9(4):443-454. [PubMed: 11983333]

10. Park S, Shin I. Fabrication of carbohydrate chips for studying protein-carbohydrate interactions. Angew Chem Int Ed Engl. 2002; 41(17):3180-3182. [PubMed: 12207382]

11. Fazio F, Bryan MC, Blixt O, Paulson JC, Wong CH. Synthesis of sugar arrays in microtiter plate. J Am Chem Soc. 2002; 124(48):14397-14402. [PubMed: 12452714]

12. Wang D, Liu S, Trummer BJ, Deng C, Wang A. Carbohydrate microarrays for the recognition of cross-reactive molecular markers of microbes and host cells. Nat Biotechnol. 2002; 20(3):275281. [PubMed: 11875429]

13. Fukui S, Feizi T, Galustian C, Lawson AM, Chai W. Oligosaccharide microarrays for highthroughput detection and specificity assignments of carbohydrate-protein interactions. Nat Biotechnol. 2002; 20(10):1011-1017. [PubMed: 12219077]

14. Willats WG, Rasmussen SE, Kristensen T, Mikkelsen JD, Knox JP. Sugar-coated microarrays: a novel slide surface for the high-throughput analysis of glycans. Proteomics. 2002; 2(12):16661671. [PubMed: 12469336]

15. Xia B, Kawar ZS, Ju T, Alvarez RA, Sachdev GP, Cummings RD. Versatile fluorescent derivatization of glycans for glycomic analysis. Nat Methods. 2005; 2(11):845-850. [PubMed: 16278655]

16. Blixt O, Head S, Mondala T, et al. Printed covalent glycan array for ligand profiling of diverse glycan binding proteins. Proc Natl Acad Sci U S A. 2004; 101(49):17033-17038. [PubMed: 15563589]

17. Rudiger H, Gabius HJ. Plant lectins: occurrence, biochemistry, functions and applications. Glycoconj J. 2001; 18(8):589-613. [PubMed: 12376725]

18. Manimala JC, Roach TA, Li Z, Gildersleeve JC. High-throughput carbohydrate microarray analysis of 24 lectins. Angew Chem Int Ed Engl. 2006; 45(22):3607-3610. [PubMed: 16639753]

19. Manimala JC, Roach TA, Li Z, Gildersleeve JC. High-throughput carbohydrate microarray profiling of 27 antibodies demonstrates widespread specificity problems. Glycobiology. 2007; 17(8):17C-23C.

20. Manimala JC, Li Z, Jain A, VedBrat S, Gildersleeve JC. Carbohydrate array analysis of anti-Tn antibodies and lectins reveals unexpected specificities: implications for diagnostic and vaccine development. Chembiochem. 2005; 6(12):2229-2241. [PubMed: 16252298] 
21. Moller I, Marcus SE, Haeger A, et al. High-throughput screening of monoclonal antibodies against plant cell wall glycans by hierarchical clustering of their carbohydrate microarray binding profiles. Glycoconj J. 2008; 25(1):37-48. [PubMed: 17629746]

22. Huang CY, Thayer DA, Chang AY, et al. Carbohydrate microarray for profiling the antibodies interacting with Globo H tumor antigen. Proc Natl Acad Sci U S A. 2006; 103(1):15-20. [PubMed: 16373501]

23. Lawrie CH, Marafioti T, Hatton CS, et al. Cancer-associated carbohydrate identification in Hodgkin's lymphoma by carbohydrate array profiling. Int J Cancer. 2006; 118(12):3161-3166. [PubMed: 16395706]

24. Wang CC, Huang YL, Ren CT, et al. Glycan microarray of Globo H and related structures for quantitative analysis of breast cancer. Proc Natl Acad Sci U S A. 2008; 105(33):11661-11666. [PubMed: 18689688]

25. Wang D, Carroll GT, Turro NJ, et al. Photogenerated glycan arrays identify immunogenic sugar moieties of Bacillus anthracis exosporium. Proteomics. 2007; 7(2):180-184. [PubMed: 17205603]

26. Blixt O, Hoffmann J, Svenson S, Norberg T. Pathogen specific carbohydrate antigen microarrays: a chip for detection of Salmonella O-antigen specific antibodies. Glycoconj J. 2008; 25(1):27-36. [PubMed: 17558551]

27. Stevens J, Blixt O, Glaser L, et al. Glycan microarray analysis of the hemagglutinins from modern and pandemic influenza viruses reveals different receptor specificities. J Mol Biol. 2006; 355(5): 1143-1155. [PubMed: 16343533]

28. Disney MD, Seeberger PH. The use of carbohydrate microarrays to study carbohydrate-cell interactions and to detect pathogens. Chem Biol. 2004; 11(12):1701-1707. [PubMed: 15610854]

29. Park S, Lee MR, Pyo SJ, Shin I. Carbohydrate chips for studying high-throughput carbohydrateprotein interactions. J Am Chem Soc. 2004; 126(15):4812-4819. [PubMed: 15080685]

30. Park S, Shin I. Carbohydrate microarrays for assaying galactosyltransferase activity. Org Lett. 2007; 9(9):1675-1678. [PubMed: 17394347]

31. Blixt O, Allin K, Bohorov O, et al. Glycan microarrays for screening sialyltransferase specificities. Glycoconj J. 2008; 25(1):59-68. [PubMed: 17914671]

32. Sharon N, Lis H. History of lectins: from hemagglutinins to biological recognition molecules. Glycobiology. 2004; 14(11):53R-62R.

33. Sharon N, Lis H. Lectins as cell recognition molecules. Science. 1989; 246(4927):227-234. [PubMed: 2552581]

34. Sharon N, Lis H. The structural basis for carbohydrate recognition by lectins. Adv Exp Med Biol. 2001; 491:1-16. [PubMed: 14533786]

35. Varki, A.; Cummings, R.; Esko, J.; Freeze, H.; Hart, G.; Marth, J. Essentials of Glycobiology. Cold Spring Harbor Laboratory Press; 1999.

36. Sharon N. Lectins: carbohydrate-specific reagents and biological recognition molecules. J Biol Chem. 2007; 282(5):2753-2764. [PubMed: 17145746]

37. Osako M, Yonezawa S, Siddiki B, et al. Immunohistochemical study of mucin carbohydrates and core proteins in human pancreatic tumors. Cancer. 1993; 71(7):2191-2199. [PubMed: 8384065]

38. Satomura Y, Sawabu N, Takemori Y, et al. Expression of various sialylated carbohydrate antigens in malignant and nonmalignant pancreatic tissues. Pancreas. 1991; 6(4):448-458. [PubMed: 1678888]

39. Shimizu K, Katoh H, Yamashita F, et al. Comparison of carbohydrate structures of serum alphafetoprotein by sequential glycosidase digestion and lectin affinity electrophoresis. Clin Chim Acta. 1996; 254(1):23-40. [PubMed: 8894307]

40. Thompson S, Cantwell BM, Cornell C, Turner GA. Abnormally-fucosylated haptoglobin: a cancer marker for tumour burden but not gross liver metastasis. Br J Cancer. 1991; 64(2):386-390. [PubMed: 1892771]

41. Okuyama N, Ide Y, Nakano M, et al. Fucosylated haptoglobin is a novel marker for pancreatic cancer: A detailed analysis of the oligosaccharide structure and a possible mechanism for fucosylation. Int J Cancer. 2005 
42. van Dijk W, Havenaar EC, Brinkman-van der Linden EC. Alpha 1-acid glycoprotein (orosomucoid): pathophysiological changes in glycosylation in relation to its function. Glycoconj J. 1995; 12(3):227-233. [PubMed: 7496136]

43. Thompson S, Guthrie D, Turner GA. Fucosylated forms of alpha-1-antitrypsin that predict unresponsiveness to chemotherapy in ovarian cancer. Br J Cancer. 1988; 58(5):589-593. [PubMed: 3265332]

44. Hirabayashi J. Lectin-based structural glycomics: glycoproteomics and glycan profiling. Glycoconj J. 2004; 21(1-2):35-40. [PubMed: 15467396]

45. Kuno A, Uchiyama N, Koseki-Kuno S, et al. Evanescent-field fluorescence-assisted lectin microarray: a new strategy for glycan profiling. Nat Methods. 2005; 2(11):851-856. [PubMed: 16278656]

46. Uchiyama N, Kuno A, Tateno H, et al. Optimization of evanescent-field fluorescence-assisted lectin microarray for high-sensitivity detection of monovalent oligosaccharides and glycoproteins. Proteomics. 2008; 8(15):3042-3050. [PubMed: 18615430]

47. Pilobello KT, Krishnamoorthy L, Slawek D, Mahal LK. Development of a lectin microarray for the rapid analysis of protein glycopatterns. Chembiochem. 2005; 6(6):985-989. [PubMed: 15798991]

48. Angeloni S, Ridet JL, Kusy N, et al. Glycoprofiling with micro-arrays of glycoconjugates and lectins. Glycobiology. 2005; 15(1):31-41. [PubMed: 15342550]

49. Koshi Y, Nakata E, Yamane H, Hamachi I. A fluorescent lectin array using supramolecular hydrogel for simple detection and pattern profiling for various glycoconjugates. J Am Chem Soc. 2006; 128(32):10413-10422. [PubMed: 16895406]

50. Pilobello KT, Slawek DE, Mahal LK. A ratiometric lectin microarray approach to analysis of the dynamic mammalian glycome. Proc Natl Acad Sci U S A. 2007; 104(28):11534-11539. [PubMed: 17606908]

51. Ebe Y, Kuno A, Uchiyama N, et al. Application of lectin microarray to crude samples: differential glycan profiling of lec mutants. J Biochem. 2006; 139(3):323-327. [PubMed: 16567396]

52. Zheng T, Peelen D, Smith LM. Lectin arrays for profiling cell surface carbohydrate expression. J Am Chem Soc. 2005; 127(28):9982-9983. [PubMed: 16011345]

53. Chen S, Zheng T, Shortreed MR, Alexander C, Smith LM. Analysis of cell surface carbohydrate expression patterns in normal and tumorigenic human breast cell lines using lectin arrays. Anal Chem. 2007; 79(15):5698-5702. [PubMed: 17580952]

54. Hsu KL, Pilobello KT, Mahal LK. Analyzing the dynamic bacterial glycome with a lectin microarray approach. Nat Chem Biol. 2006; 2(3):153-157. [PubMed: 16462751]

55. Tateno H, Uchiyama N, Kuno A, et al. A novel strategy for mammalian cell surface glycome profiling using lectin microarray. Glycobiology. 2007; 17(10):1138-1146. [PubMed: 17693441]

56. Tao SC, Li Y, Zhou J, et al. Lectin microarrays identify cell-specific and functionally significant cell surface glycan markers. Glycobiology. 2008; 18(10):761-769. [PubMed: 18625848]

57. Haab BB, Dunham MJ, Brown PO. Protein microarrays for highly parallel detection and quantitation of specific proteins and antibodies in complex solutions. Genome Biol. 2001; 2(2) RESEARCH0004.

58. Kjeldsen T, Clausen H, Hirohashi S, Ogawa T, Iijima H, Hakomori S. Preparation and characterization of monoclonal antibodies directed to the tumor-associated O-linked sialosyl-2----6 alpha-N-acetylgalactosaminyl (sialosyl-Tn) epitope. Cancer Res. 1988; 48(8):2214-2220. [PubMed: 2450649]

59. Hanisch FG, Hanski C, Hasegawa A. Sialyl Lewis(x) antigen as defined by monoclonal antibody AM-3 is a marker of dysplasia in the colonic adenoma-carcinoma sequence. Cancer Res. 1992; 52(11):3138-3144. [PubMed: 1350509]

60. Thompson S, Stappenbeck R, Turner GA. A multiwell lectin-binding assay using lotus tetragonolobus for measuring different glycosylated forms of haptoglobin. Clin Chim Acta. 1989; 180(3):277-284. [PubMed: 2743580]

61. Chen S, LaRoche T, Hamelinck D, et al. Multiplexed analysis of glycan variation on native proteins captured by antibody microarrays. Nat Methods. 2007; 4(5):437-444. [PubMed: 17417647] 
62. Forrester S, Kuick R, Hung KE, Kucherlapati R, Haab BB. Low-volume, high-throughput sandwich immunoassays for profiling plasma proteins in mice: identification of early-stage systemic inflammation in a mouse model of intestinal cancer. Molecular Oncology. 2007 in press.

63. Orchekowski R, Hamelinck D, Li L, et al. Antibody microarray profiling reveals individual and combined serum proteins associated with pancreatic cancer. Cancer Res. 2005; 65(23):1119311202. [PubMed: 16322270]

64. Gao WM, Kuick R, Orchekowski RP, et al. Distinctive serum protein profiles involving abundant proteins in lung cancer patients based upon antibody microarray analysis. BMC Cancer. 2005; 5:110. [PubMed: 16117833]

65. Patwa TH, Zhao J, Anderson MA, Simeone DM, Lubman DM. Screening of glycosylation patterns in serum using natural glycoprotein microarrays and multi-lectin fluorescence detection. Anal Chem. 2006; 78(18):6411-6421. [PubMed: 16970316]

66. Zhao J, Patwa TH, Qiu W, et al. Glycoprotein microarrays with multi-lectin detection: unique lectin binding patterns as a tool for classifying normal, chronic pancreatitis and pancreatic cancer sera. J Proteome Res. 2007; 6(5):1864-1874. [PubMed: 17428079] 

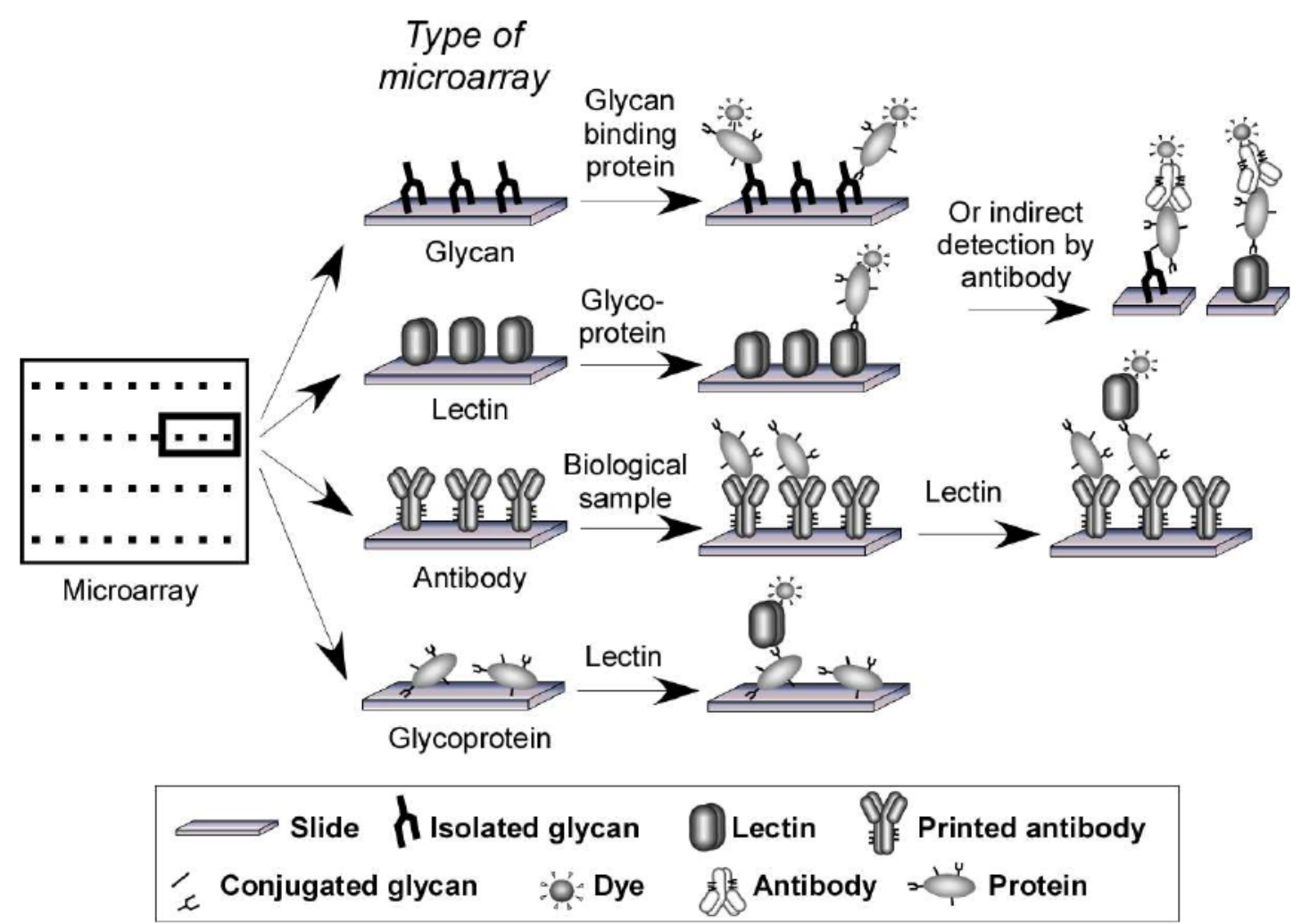

Figure 1.

Microarray formats for glycoproteomics research. The types of microarrays depicted are glycan arrays, lectin arrays, antibody-lectin sandwich arrays, and glycoprotein arrays. A detection strategy using a fluorescent dye is depicted, although other detection methods could be used, such as surface-plasmon resonance or chemiluminescence. Glycoprotein arrays, involving the isolation of glycoproteins from biological samples using chromatographic methods, followed by the probing by lectins of the glycans microarrayed proteins $[65,66]$, are not discussed in the text but are depicted here for completeness. 


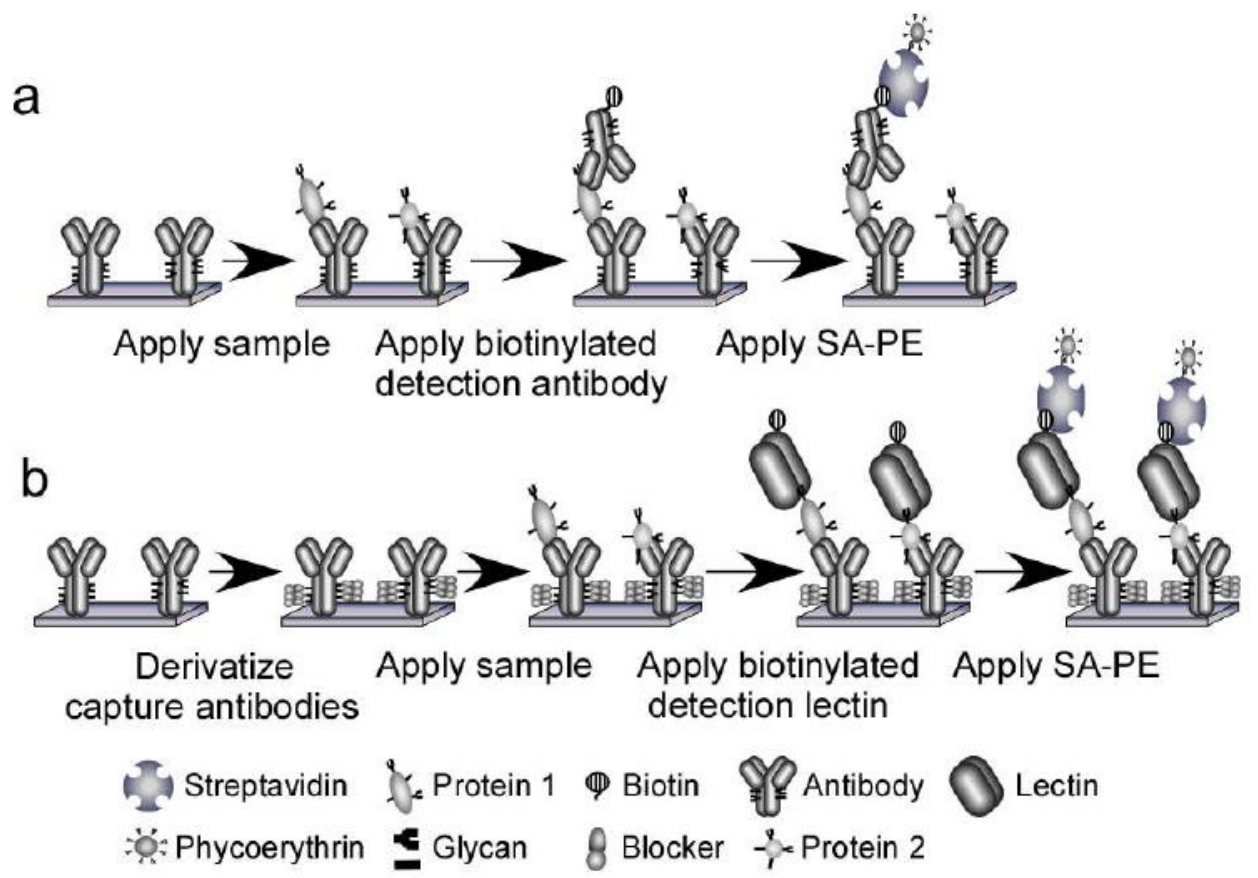

C

Sample:

Protein detection

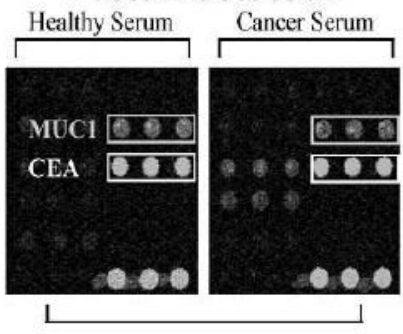

Detection: Anti-Pan-CEACAM \& Anti-MUCl
Glycan detection

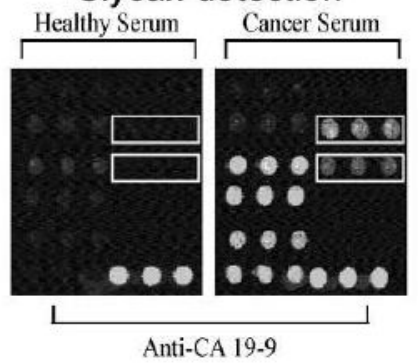

Figure 2.

Complementary detection of protein levels and glycan levels on captured proteins using antibody arrays. a) Array-based sandwich assays for protein detection. Multiple antibodies are immobilized on a planar support, and the captured proteins are probed using biotinylated detection antibodies, followed by fluorescence detection using phycoerythrin-labeled streptavidin. b) Glycan detection on antibody arrays. This format is similar to above, but the detection reagents target the glycans on the captured proteins rather than the core proteins. The glycans on the immobilized antibodies are chemically derivatized to prevent lectin binding to those glycans. c) Detection of differential glycosylation. A healthy patient serum sample and a cancer patient serum sample were incubated on each pair of arrays (same two samples in each pair). The boxes indicate the capture antibodies targeting MUC1 and CEA. The arrays were detected using either a mixture of two antibodies to detect the MUC1 and CEA core proteins (left array in each pair) or an antibody detecting the CA 19-9 carbohydrate epitope (right array in each pair). The data show equivalent core protein levels between the healthy and cancer serum, but high levels of the CA 19-9 glycan in the cancer serum. The other spots showing signal are control proteins or other proteins containing the CA 19-9 epitope. 

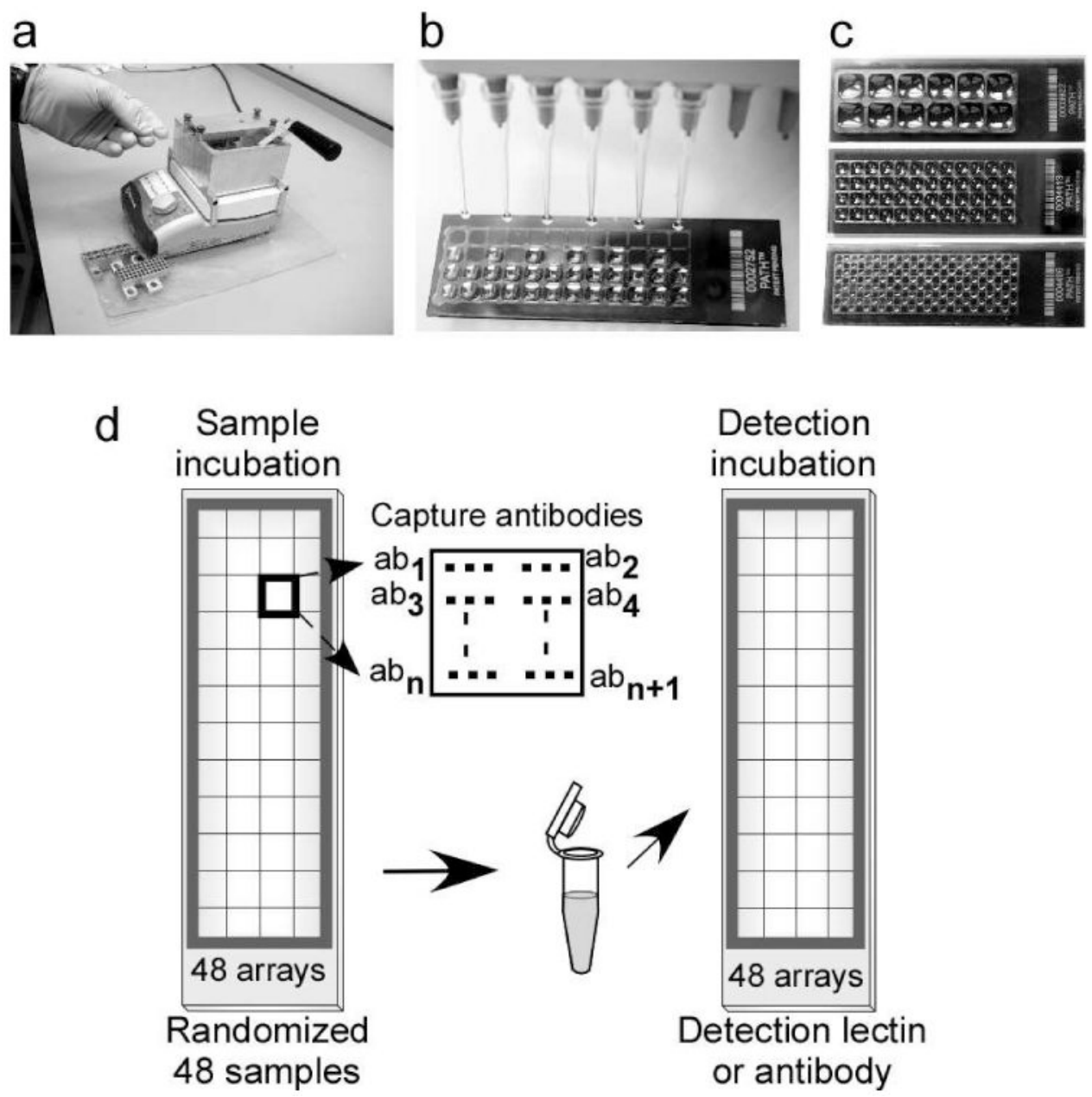

Figure 3.

Practical, high-throughput processing of antibody microarrays. a) Imprinting hydrophobic boundaries. Wax is melted by the hotplate under the bath, and a slide is inserted upsidedown into the holder. Bringing the lever forward raises a stamp out of the wax bath to touch the slide, imprinting the design onto the slide to form borders around multiple arrays. Two stamps are shown in front of the machine. b) Loading samples onto a slide containing 48 arrays. The arrays are spaced by $4.5 \mathrm{~mm}$, which is compatible with the $9 \mathrm{~mm}$ spacing of standard multichannel pipettes. c) Samples loaded onto slides containing 12 (top), 48 (middle), and 192 (bottom) arrays (96 samples loaded). d) Strategy for profiling glycans in multiple samples. Forty-eight or sixty identical microarrays are printed on one microscope slide, segregated by hydrophobic boundaries. A set of serum samples is incubated on the arrays in a random order, and each slide is probed with a single antibody or lectin. 